\title{
ACC

\section{El secreto profesional en el cuidado enfermero}

\section{Hernández Castro Vidalina Elvira ${ }^{1}$, Del Río Perales Erika Diana ${ }^{2}$, Carrasco Navarrete Mary Susan ${ }^{3}$}

\section{INFORMACIÓN DEL ARTÍCULO}

Historia del artículo:

Recibido el 5 de junio de 2016

Aceptado el 5 de octubre de 2016

\section{Palabras claves:}

Secreto Profesional

Enfermería

Cuidado

\section{RESUMEN}

El profesional de enfermería en su cotidiano se enfrenta a numerosas situaciones, en los que se evidencia principios y dilemas éticos en el contexto del secreto profesional. La presente investigación, tuvo como objetivo identificar y analizar cómo se practica el secreto profesional en el cuidado enfermero. Es una investigación cualitativa con abordaje metodológico de Estudio de caso. El escenario fue el Hospital Provincial Docente Belén Lambayeque y los sujetos de estudio estuvo conformado por 06 enfermeras asistenciales que laboran en los servicios de Ginecología, Medicina y Cirugía. En el marco teórico en relación al secreto profesional se consideró a Pastor García, León Correa y Vielva Asenjo. Respecto al cuidado se sustentó con los autores Mingote A., Potter P, Waldow R y Kérouac S. Se recogieron los datos con entrevista semiestructurada a profundidad, previo consentimiento informado: Recurriéndose al análisis de contenido emergieron las categorías: Confidencialidad o divulgación de lo que me dice el paciente; Secreto profesional principio bioético en enfermería; y Escaso conocimiento de documentos éticos legales, que dificultan la práctica del secreto profesional.

\footnotetext{
${ }^{1}$ Licenciada en enfermería. Enfermera del Centro de Salud San Martin- Lambayeque, Perú. Email: vielhc21@hotmail.com

2 Licenciada en Enfermería. Enfermera con trabajo independiente. Chiclayo, Perú.

3 Magister en Bioética. Docente en la Escuela de Enfermería de la Universidad Católica Santo Toribio de Mogrovejo. Enfermera asistencial del Servicio de UCI del Hospital Regional Docente las Mercedes. Chiclayo, Perú. Email: mcarrasco@usat.edu.pe
} 


\section{Professional secrecy in nursing care}

\section{ABSTRACT}

Keywords:

Professional secrecy Nursing

Care
The professional nurse in her daily life is faced with many situations, in which evidence principles and ethical dilemmas in the context of profesional secrecy. This research study, had as objective to identify and analyze how to practice the profesional secrecy in the nursing care. It is a type of qualitative research with methodological approach of case study, supported by Rodriguez Gomez G.; Bernal Torres. The scenario was the Provincial Teaching Hospital Bethlehem Lambayeque and the subject of study was composed of 9 nurses working in the Gynecology Service, Medicine and Surgery. The theoretical conceptual framework was based on various authors, in relation to the professional secrecy, it was considered to Pastor Garcia and Leon Belt ; Vielva Asenjo J. On the other hand focusing on nursing and care support with the authors Mingote Adam; Potter P.; Waldow R.; Kerouac S. It was used as data collection instruments the semistructured interview to depth, prior and informed consent for the information obtained has been used the content analysis of where the categories emerged: Confidentiality or disclosure of what I said the patient; professional secrecy bioethical principle in nursing; and little knowledge of ethical legal documents, which make it difficult the practice of professional secrecy. It took into account the ethical criteria and scientific rigor in the development of the present investigation.

\section{Introducción}

El secreto profesional es un tema muy polémico en la actualidad, pues concierne prácticamente a todo profesional a quien, directa o indirectamente, se hacen de su conocimiento hechos o acciones que guardan una infinidad de información personal e íntima de la persona hospitalizada, cuya revelación tiene consecuencias graves no sólo para el profesional de enfermería que comete la falta, sino que pondría en tela de juicio a la profesión y el hospital o institución; creándose conflictos, desconfianza, y a su vez, una responsabilidad moral y legal que puede ser causa de una demanda por daños y perjuicios a la persona misma o a sus familiares, comprometiéndose su dignidad y el respeto a su autonomía. ${ }^{1}$
Siguiendo la trayectoria histórica, tenemos que desde la antigüedad el Secreto Profesional en el ejercicio de la medicina, quedaba protegida por el famoso juramento de Hipócrates (460 - 377 a. c), que ha conservado su valor a lo largo de la historia; donde afirma "Mi boca no dará a conocer lo que mis ojos hayan visto o lo que mis oídos hubieran percibido, mi lengua callará los secretos que me sean confiados. ${ }^{2}$ Con respecto a Enfermería es importante resaltar que una de las pioneras Florence Nightingale, contribuyó en la elaboración

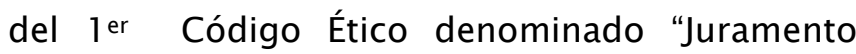
Nightingale" redactado en 1893, que Dice: Consideraré como confidencial toda información que me sea revelada en el ejercicio de mi profesión, así como todos los asuntos familiares de mis pacientes. ${ }^{3}$ 
Parece que en la actualidad la enfermera(o) ha olvidado el juramento antes mencionado; es por ello la necesidad de continuar fomentando por generación, esa imagen de confianza que tiene la sociedad hacia ese gran número de hombres y mujeres, que dedican su vida a ayudar a la persona sana o enferma. 1 Por ello el secreto profesional en enfermería, surge de la evolución de normas éticas y deontológicas que amparan y protegen al secreto profesional, encontrándose a nivel Internacional, el Código Deontológico del Consejo Internacional de Enfermeras (CIE). En nuestro país las Enfermeras, cuentan con el Código de Ética y Deontología del Colegio de Enfermeros del Perú; asimismo, la Ley del Trabajo de la Enfermera(o) $N^{\circ} 27669$; la Ley General de Salud N²6842; la Constitución Política del Perú y el Código Penal Peruano.

En este sentido, el secreto profesional en enfermería, está amparado bajo normas éticas y deontológicas, mencionadas anteriormente, siendo primordial conocerlas y llevarlas a la práctica; pero es una realidad, que existen enfermeras(os) que desconocen los documentos éticos legales que respaldan este secreto y ante tales circunstancias, aún la Enfermera no se detiene a pensar, sobre las consecuencias de su irresponsabilidad, ante la divulgación de información, obtenida en el ejercicio de su profesión, quebrantando así el juramento de Florencia Nightingale y a su ética profesional.

Por ello, cabe mencionar que durante la formación de pregrado y considerando las experiencias de las investigadoras en las prácticas clínicas, realizadas en los diferentes servicios de hospitalización, se Evidenció situaciones en que algunas(os) enfermeras (os), no toman en cuenta la confidencialidad y la lealtad que le deben a sus pacientes durante el tiempo que dura su estancia hospitalaria; se observó en algunos casos, que durante el reporte de enfermería en cada turno, las enfermeras expresan algunas frases a sus compañeras de trabajo "...tengan cuidado con el paciente de la cama número 4 , porque es código rojo... además, pocas veces vienen a visitarlo sus familiares..." y en muchas ocasiones, estos comentarios los realizan en presencia de los estudiantes de enfermería de las diferentes universidades y en presencia de otro personal de salud. En el mismo sentido, expresaron otros comentarios que reflejan el incumplimiento del secreto profesional como "... el paciente de la cama número 6 ingresó por intento de suicidio”; “... saben el paciente del ambiente privado está irritado, tengan cuidado las puede lastimar, tiene SIDA...". En otras ocasiones se escucha comentarios entre enfermeras, en los pasadizos de los hospitales "...esa chica es problemática y de todo se queja, tengan cuidado con ella... dicen que ella ingresó borracha a la casa de su enamorado por la ventana y al momento de darse cuenta la madre... la empujó desde el cuarto piso... "ella al momento de caer se cogió de los muros de las ventanas y recién en el segundo piso se arrojó, por eso no tiene lesiones graves; pero está muy ansiosa"...

Por otro lado, algunos familiares de otros pacientes preguntaron en el momento de la visita... ¿Por qué el paciente está sujetado de pies y manos en la baranda de la cama? y la enfermera responde en tono alto "... el paciente tiene SIDA". Estos comentarios se difundieron hasta otros profesionales del equipo de salud y del personal técnico de enfermería, producto de lo expresado por algunas(os) enfermeras(os).

En este sentido, también se observó que ciertos profesionales de enfermería, hacen caso omiso a la decisión de la persona e ignoran las consecuencias, que puede conllevar su irresponsabilidad, ante la privacidad de los día gnósticos y/o confidencialidad, divulgado ésta información dentro y fuera de su trabajo, quebrantando la confianza y los derechos que tienen los pacientes, que se encuentran bajo su cuidado.

Lo expresado anteriormente como problemática, ocasiona daño tanto moral, psicológico y físico, no solo al paciente, sino también a su familia e 
inclusive produciendo en algunas ocasiones rechazo de la sociedad. Estas situaciones repercuten también en los estudiantes de enfermería en formación, porque asumen el mismo posicionamiento y actitud cuando se insertan en el campo laboral, como parte de las vivencias obtenidas durante todo el desarrollo de su formación de pregrado. Esto se evidencia, en el comportamiento que tienen algunos estudiantes en las aulas universitarias, los cuales divulgan situaciones personales y familiares de sus compañeros de clase.

Ante la problemática expuesta, las investigadoras se plantearon la siguiente interrogante ¿Cómo se practica el secreto profesional en el cuidado enfermero? y tuvo como objetivo identificar $y$ analizar cómo se practica el secreto profesional en el cuidado enfermero.

\section{Metodología}

La presente investigación fue de tipo cualitativa y según A. Moreno4 consiste en descripciones detalladas de situaciones, eventos, personas, interacciones y comportamientos que son observables; además, incorpora lo que los participantes dicen, sus experiencias, actitudes, creencias, pensamientos y reflexiones, tal como son expresados por ellos mismos; el mismo autor lo considera como un proceso activo, sistemático y riguroso de indagación dirigida, en el cual se toman decisiones sobre lo investigable, en tanto se está en el objeto de estudio; por lo tanto están orientados a descubrir el sentido y significado de las acciones sociales.

El escenario fue el Hospital Provincial Docente Belén Lambayeque, que pertenece al Ministerio de Salud; tiene la categoría Hospital II - 1; brinda a la población servicios de hospitalización, consulta externa, emergencia, programas preventivos promocionales, farmacia, ayuda diagnóstica como rayos $X$ y laboratorio. Además es un establecimiento de referencia para la provincia de
Lambayeque y algunas provincias de la Región macro norte.

Para esta investigación se utilizó el estudio de caso, permitiendo comprender las diversas características de los sujetos de estudio; el cual se hizo de manera narrativa, valiéndose de un amplio marco teórico, haciéndose uso del pensamiento crítico. Los sujetos de estudio estuvieron conformados por 06 enfermeras asistenciales que laboran en el Hospital Provincial Docente Belén Lambayeque, teniendo como criterios de inclusión: enfermeras asistenciales de los servicios de ginecología, medicina y cirugía; con tiempo de 3 años en el servicio; según condición laboral nombradas y contratadas. Para la obtención de datos se hizo uso de una entrevista semiestructurada a profundidad, en confianza y respetándose los principios éticos de autonomía, privacidad y confidencialidad.

Durante las entrevistas se hizo uso de una grabadora, con una duración de 25 a 35 minutos cada entrevista. Sin embargo, algunas enfermeras no aceptaron participar en el estudio, al conocer que sus entrevistas serían grabadas y finalmente se evidenció algunos casos, donde se postergó la entrevista ya planificada, acordando nueva fecha para la realización de la misma, adecuando al horario de cada una de las entrevistadas y teniendo en consideración su disponibilidad de tiempo, sin interferir en sus funciones, siendo mayormente en horario nocturno.

Los datos recolectados se interpretaron mediante el análisis de contenido, el cual cuenta con tres etapas 5 :

Primera etapa: Pre análisis, esta es la etapa de organización del material a analizar en el que se diseña y define los ejes del plan que permitieron examinar los datos y realizar efectivamente el análisis del contenido. La segunda etapa tenemos la codificación, que consiste en realizar una transformación de los "datos brutos" (el material original) a los "datos útiles". En la tercera etapa, la categorización, se realizó la organización y 
clasificación de las unidades obtenidas en base a criterios de diferenciación. Durante el desarrollo de la investigación se aplicó los principios de la bioética personalista de Sgreccia6, donde se enfatizó el respeto a la dignidad de las personas, a los derechos humanos, así como la confidencialidad, verdad, sinceridad y la lealtad.

\section{Resultados, análisis y discusión}

Después de realizar las entrevistas semiestructuradas a profundidad a los sujetos en estudio, se realizó el análisis de datos y se obtuvo tres categorías que a continuación se detallan:

Categoría I.- Confidencialidad o divulgación de lo que me dice el paciente.

Categoría II.- Secreto profesional principio bioético en enfermería.

Categoría III.- Escaso conocimiento de documentos éticos legales, que dificultan la práctica del secreto profesional.

\section{Categoría I. Confidencia o divulgación de lo que me dice el Paciente.}

El término de confidencialidad significa "lo que se hace o dice en confianza o con seguridad recíproca entre dos o más personas" 8. Ante lo expuesto y comparándolo con la información obtenida a través de las entrevistas, las enfermeras manifiestan lo siguiente:

"El secreto profesional es algo que tengo que guardar; una confidencia de lo que me dice el paciente cuando tiene algún problema, ellas no quieren que el resto se enteren. Es por eso que recuren a la enfermera y nos cuentan sus cosas."(Géminis- $A$ )

"El secreto profesional es ser confidente de la persona, es decir que el paciente nos informa algo íntimo de él." (Virgo-A)
"El secreto profesional es algo que debo guardar, callar ante lo que me dice el paciente." (Piscis-A)

A través de los discursos, las enfermeras consideran que en el ejercicio de su profesión se ven comprometidas en la vida íntima de sus pacientes, ante la diversidad de confesiones que son facilitados de manera voluntaria, y a la vez es la enfermera quien está comprometida a callar o guardar dicho aspecto de intimidad, que está implícito en el compromiso profesional, donde debemos preservar la confidencialidad, porque su violación hace que las personas no proporcionen información a los profesionales.

Por otro lado, el profesional de enfermería, se puede ver obligado a revelar el secreto profesional en diversas circunstancias: Cuando se atienda a personas que puedan haber sido víctimas de un delito; en caso de mala praxis; cuando se pone en riesgo la vida o la salud del propio paciente: se da este caso, por ejemplo, cuando un enfermo o una paciente nos manifiesta idea de suicidio; para evitar un daño a otras personas, durante el ejercicio de la justicia y el bien general de la sociedad: de ahí que se haya reconocido la obligación de declarar enfermedades infecciosas o trastornos mentales que signifiquen un peligro social por ello se declaran los malos tratos, o las heridas producidas en posibles delitos, y se colabora con la justicia cuando el juez lo exige; ante los que estaría justificado revelar el secreto.3,8

En el caso de los estudiantes de enfermería, forman parte del equipo de salud, y también se ven afectados por la obligación del secreto compartido. Por ello es importante que durante la formación académica de pregrado, se fortalezca el componente ético debiendo tener la obligación de guardar el secreto, evitando su violación, aunque aún no hayan requerido el carácter de profesionales. Como lo expresan los discursos:

“... Otro caso cuando está en riesgo la vida de la paciente donde tiene bastante sangrados, tanto enmarca la vida de la paciente, en ese caso se tiene 
que informarse a los familiares $y$ de ese modo romper el secreto profesional." (Géminis-D)

“...Es importante que la enfermera guarde el secreto profesional siempre y cuando no afecte a otras personas, por ejemplo a su familia o a nosotros mismos..". (Virgo-B)

“...Un adolescente me cuenta que su enamorada lo ha dejado y que Él tiene intentos de querer auto eliminarse, yo tengo que informar eso, porque corre en riesgo su vida..." (Virgo-B)

“...No sé cómo vino acá una sobrina estudiante de enfermería, y comentamos entonces: Ella me dice "señora, el señor ha comentado y también su familia que tiene un hijo que ha fallecido de SIDA. No le dijo eso". No, no ha comentado nada y le pregunte ¿en qué ha trabajado el señor?; trabajaba recogiendo desechos de basura y su hijo también trabajaba igual. ¿Pero cómo Él se contagió? por una aguja que encontró en la basura". Entonces de donde sabemos, que el señor que tiene el mismo trabajo le haya sucedido igual; pero me dijo "señora, nadie sabe".

Yo informe al interno, al médico lo sucedido para que averigüen, investiguen, y hagan un estudio al paciente. (Virgo-C)

Como se observa en los discursos, las enfermeras durante su desempeño laboral, obtienen informaciones y confidencias de los pacientes a través del dialogo que se da entre ambos, donde se comprometen a mantener oculta dicha información; pero ante situaciones que ponen en riesgo la vida del paciente, familiares y del equipo de salud; se siente en la obligación de revelar lo confiado, evitando daños mayores, por ello la confidencialidad pide que el profesional enfermero debe respetar el secreto profesional, y que sea cuidadoso a la hora de compartir información con los miembros del equipo. Por eso hay que recordar que "tan sólo es materia de comunicación aquello que interese dar a conocer para la salud del paciente y tan sólo a los que puedan ayudar". Por ello la protección de la confidencialidad es un deber de la institución, responsable de garantizar la protección de los datos frente a un mal uso o acceso injustificado a los mismos. 8

Por otro lado, la persona en situación de problemas de salud ofrece al cuidador información sobre su propio cuerpo, sus pensamientos, su vida. Si se hace pública la información, se rompe el vínculo de relación establecido, lo que supone una violación de la privacidad y una falta de respeto a la condición de ser humano. 9 En muchas ocasiones se divulga la información obtenida de documentos legales como la historia clínica, como también lo confiado por el paciente; revelando de esta manera el secreto profesional, haciéndose pública la información y a la vez causando daño a persona y/o quebrantando la confianza6. Tal como se afirma en el siguiente discurso:

“...El vigilante no tiene por qué dejar pasar a personas que solo vienen a enterarse, y luego se inicia el rumor, lo cual origina una distorsión en la información. Tampoco no debería divulgar cualquier diagnóstico de la persona hospitalizada; esto también involucra a los técnicos". (Piscis-B)

Por eso las enfermeras deducen que la divulgación de los diagnósticos de los pacientes, es mal informado por el personal técnico de enfermería, a personas ajenas al servicio, originando que malinterpretan los diagnósticos produciendo de este modo, malos entendidos; concluyendo que no es la enfermera quien rompe el secreto profesional con respecto al cuidado del paciente. Es aquí donde el profesional, depositario de la información privada entregada por el paciente con un fin específico, no cumple con este compromiso, la confianza se romperá obstaculizando el correcto diagnóstico y por ende el pronóstico de la enfermedad y de esta manera dificultando la confidencialidad y protección de la información. Desde este punto de vista, los exámenes de laboratorio deben ser considerados parte del secreto profesional y nadie que no sea el paciente puede tener acceso a ellos sin su previa 
autorización, a menos que esté impedido por razones de salud en forma temporal o definitiva. ${ }^{10}$ Además durante el reporte de enfermería, el informe sobre el estado del paciente, debe llevarse a cabo en privado, donde sea improbable que puedan ser escuchadas por casualidad ${ }^{11}$ evitando de esta manera ir contra la dignidad del ser humano, por ello se formuló la segunda categoría.

\section{Categoría II. Secreto Profesional, principio bioético en Enfermería.}

Desde el momento en que la enfermera cuida al enfermo, surge automáticamente una obligación de secreto, sobre todas aquellas cosas que se llegue a conocer en relación profesional. La relación entre enfermera- paciente se establece sobre la base de confianza. Parte de esa confianza la constituye la obligación de guardar silencio de aquellas cosas que el paciente comunique a la enfermera. Por ello, el secreto se define como el deber de no divulgar los que, debido a razón de justicia, fidelidad, amistad, etc., quiere que se mantenga oculto. ${ }^{2}$

A lo anterior mencionado se agrega la bioética, que es el estudio sistemático de la conducta humana desde las perspectivas de las ciencias de la vida y de la salud, analizada a la luz de los valores y principios morales. A su vez tiene por finalidad el análisis racional de los problemas morales ligados a la biomedicina y de su vinculación con el ámbito de derecho y de las ciencias humanas 12 .

Es relevante mencionar que el secreto obliga a todos los profesionales sanitarios y también en aquellos otros profesionales que, sin ser sanitarios, colaboran con éstos $y$, por sus funciones, tienen igual acceso a información de carácter confidencial, como son los administrativos o los técnicos de informática, e incluso estudiantes de medicina o enfermería que hacen prácticas en el hospital, estando sujetos a una obligación de silencio ${ }^{2,3}$. tal como es expresado en los siguientes discursos:
"El secreto profesional es una obligación ética de información que hay que manejarla con mucho cuidado y absoluta cautela sobre algún paciente, como en el caso de nosotros que trabajamos con el sector salud." (Virginia Henderson-A)

"Al hablar del secreto profesional está ligado mucho a la ética; a la formación; a los valores morales como la responsabilidad, solidaridad; y a los principios éticos por ejemplo la justicia y la verdad..." (Escorpio-A)

Los sujetos de estudio, de acuerdo a su formación ética, salvaguardan con absoluta reserva todo lo confidencial con sus pacientes, siendo responsable de sus actos. De ahí, en lo que al secreto profesional enfermero se refiere, que se reconozca bajo su ámbito todo aquello que forma parte de las personas y su entorno, en tanto éstas no lo quieran revelar o no autoricen su revelación, o su divulgación pueda producirles cualquier menoscabo en su dignidad, libertad o intimidad.

Del mismo modo, hay que recordar también que la dignidad de la persona no acaba en sí misma, ni siquiera con su propia vida, ya que incluso después de la vida, sigue siendo preciso mantener la dignidad de aquella en el recuerdo, así como la de su propia familia, porque la memoria del difunto exige igualmente respeto al ámbito privado. La muerte del otro no nos da derecho a abrir las puertas de su intimidad. ${ }^{3}$ Por ello, el secreto de la vida privada es un derecho inalienable de toda persona, y la enfermera tiene la obligación esencial de considerar como confidencial todos los datos que posee sobre sus pacientes, cualquiera que sea la fuente de información.

A su vez, el profesional de enfermería es el responsable absoluto de todos los daños que cause sus errores consientes plenamente y está obligado a responder por sus actos, ya que lleva implícito la libertad de actuar o no como sujeto de mérito (de bien y ser consecuente en el trabajo, hacer un buen uso de cada principio ético, de guiarse por el camino de la moral y ser cuidadoso 
en las cosas que son puestas a su cuidado). Se deberá mantener el secreto de su actuación aún después de la muerte de los usuarios que haya cuidado, evitando de esta forma perjuicio a terceros. ${ }^{1}$ Así como se expresa en los siguientes discursos:

“...Cuando ingresa una paciente, el trabajador de emergencia la reconoce como familiar de algún compañero de trabajo $y$ esta paciente es hospitalizado en este servicio para su procedimiento que en este caso es un aborto en curso; luego las colegas llegan a preguntar cuál es el motivo de ingreso de la paciente, pero somos nosotras las enfermeras quienes tenemos que proteger la integridad de la paciente, por ello no comentamos nada y preferimos decirles otras cosas a las colegas que vienen a preguntar a este servicio..." (Géminis-B)

Las enfermeras nos reflejan que la infidencia de diagnósticos no es por el personal de enfermería del servicio, sino por parte de los profesionales de enfermería de otros servicios; por tal motivo la enfermera salvaguarda la integridad del paciente, no relatando el diagnóstico real.

Uno de los aspectos más relevantes en enfermería, es la práctica de valores éticos, que se traduce en su comportamiento. La enfermera no solo ejerce una carrera, una materia, o una misión, sino también una función y una actitud libremente escogida, lo que implica estar presente en su globalidad a nivel personal y profesional. ${ }^{6}$ En tal sentido, la ética de valores propone una ética basada en la defensa del hombre y de la dignidad de la persona humana, y se fundamenta con la verdad13, Reflejándose en el siguiente discurso:

“... Estuve en una cirugía de AQV y resulta que entra una paciente para ligaduras de trompas, pero el ginecólogo nunca encontró una trompa, así fue; entonces me vi obligada en romper con el secreto profesional en el aspecto de que tuve, que decirle la verdad a la paciente... Fue un gran dilema ético que a veces los profesionales nos vemos en ese momento comprometidos..."

\section{(Virginia Henderson-C)}

Igualmente la enfermera al verse involucrada en dicha acción en la que está de por medio sus valores éticos o en ocultar un procedimiento no realizado por el especialista; la enfermera se siente en la obligación de decir la verdad a la paciente evitando consecuencias posteriores que perjudiquen a la misma. Esta es una situación en la que emergió un dilema ético, que muchas veces se origina en nuestro actuar profesional diario y que dificulta una adecuada toma de decisiones.

Por lo tanto, en los dilemas éticos se plantea una situación posible en el ámbito de la realidad pero conflictiva a nivel moral, y se solicita una solución razonada del conflicto, o un análisis de la solución.

Los dilemas pueden ser hipotéticos, cuando se plantean problemas abstractos, generales, que a veces son de difícil ubicación en la realidad, pero quienes los analizan reconocen que son siempre posibles de presentarse en determinadas ocasiones de la vida real ${ }^{14}$. Como se expresan en los siguientes discursos:

“...En la mayoría de veces nos hemos encontrado con dilemas éticos, donde se involucra el secreto profesional, por ejemplo personas jóvenes, adultos maduros, entre ellos varones nos piden que por favor no informemos sus diagnósticos a sus familiares más allegados, como son las esposas, o parejas..." (Claudiana-B)

“...Nos enfrentamos ante un dilema, en el sentido de que tenemos que regirnos al principio de beneficencia enfocado en las personas, como en este caso a las esposas o sus parejas, informándoles del diagnóstico para que tomen las medidas de prevención..."(Claudiana-B)

“... Hay casos que en principio de la enfermedad los pacientes cambian su personalidad, siendo egoístas, o si no tratan de decir porque a mí y porque no a otras personas y se cierran en esa negatividad, entonces ellos están así como ambivalentes, en querer proteger y querer hacer 
daño a otras personas, así como se le hicieron quizás a ellos mismos. Esta es una situación en la cual se presenta dilemas de decisión de las enfermeras para poder conservar o no el secreto profesional." (Claudiana-B)

“...Siempre van a pasar problemas de dilemas éticos, una serie de oposiciones contra otro principio, es allí donde el equipo de salud debemos estar preparados para ese tipo de problemas, entiendo un trabajo organizado y de esta manera no afecte al paciente hospitalizado ni al personal de salud." (Claudiana-F)

Cabe mencionar que durante el ejercicio laboral las enfermeras son quienes están de manera constante con el paciente y esto permite tener una comunicación terapéutica permitiéndole enterarse más a fondo de la vida personal del ser cuidado, evidenciándose diagnósticos que pueden acarrear daños a terceras personas, donde los pacientes no quieren que su diagnóstico sea revelado a sus familiares o están en situaciones conflictivas internamente cuyo fin es causar daño; es allí donde la enfermera se encuentra ante un dilema ético, en la cual tiene que utilizar su pensamiento crítico y reflexivo para tomar una decisión, en la que no ponga en peligro la vida del familiar ni del personal de salud; por ello se siente comprometida en comunicar el diagnóstico, brindando una mejor atención de salud y que el familiar directo del paciente tome las medidas preventivas correspondiente.

Actualmente en toda relación sanitaria se presentan principios bioéticos ${ }^{6}$, como es el de beneficencia entendida como un hacer el bien, de forma integral al sujeto y respetando su libertad; otro de los principios es el de justicia se enfoca se refiere a lo que uno merece, lo que es justo o correcto. Las personas merecen trato amable y no deben ser discriminadas por razones sociales o de capacidad mental; también el principio de autonomía se refiere a la autodeterminación, libertad de elección de un paciente competente; y por último el principio de no maleficencia que se enfoca a no hacer daño, además no producir lesiones en la integridad del paciente que no sean las estrictamente terapéuticas.

No hay que olvidar que primero es hacer el bien y luego evitar el mal y que tanto uno como otro se miden por el bien que se deja de hacer o del que se priva voluntariamente o el bien que se conserva al no hacer el mal. Por ello, los principios ayudan a solucionar los actos desde la responsabilidad, ya que desde ellos se responde a las situaciones y a las personas concretas. Esto se enriquece a través de los documentos éticos legales que respaldan y norman el secreto profesional, por el cual se efectuó la siguiente categorización:

\section{Categoría III. Es caso conocimiento de documentos ético-legales, que dificultan la práctica del secreto profesional.}

Los códigos deontológicos son el resultado de una larga tarea de selección de normas y criterios mediante los cuales los profesionales regulan la práctica de su profesión. ${ }^{8}$ Además es una lista escrita de valores profesionales y normas de conducta, que proporciona un marco de referencias para tomar decisiones y exige revisión periódica que refleja los cambios en la sociedad y en la profesión'

Es así que los documentos éticos legales ayudan, a que el personal profesional debe tener acceso a la información derivaba de la relación terapéutica y para llevar a cabo unos cuidados de enfermería de calidad, se hacen necesarios los códigos de ética y deontología; por ende, estos son respaldados por la Ley General de Salud N²6842; la Constitución Política del Perú; Código Deontológico del Consejo Internacional de Enfermeras (CIE); el Código Penal Peruano; Código de Ética y Deontología del Colegio de Enfermeros del Perú; y la Ley del Trabajo de la Enfermera(o) $\mathrm{N}^{\circ}$ 27669. Estos documentos normativos limitan el uso de la informática para garantizar el honor y la intimidad personal y familiar de los ciudadanos y el pleno ejercicio de sus derechos; y se convierte en una herramienta de trabajo útil, fundamental $y$ necesario en el 
contexto profesional actual. 6 Como se expresa en los discursos:

"Solo conozco la historia clínica, tenemos el Código Internacional de Ética Médica; el Código de Ética y Deontología del Colegio Enfermeros del Perú y el Juramento Hipocrático, ya no recuerdo otro." (Virginia Henderson-E)

"El consentimiento informado, el cual nos permite tener la autorización del paciente, para poder dar información o no todo respecto a Él. No conozco nada más." (Claudiana-E)

"Bueno no conozco documentos; legal, es la historia clínica que es manipulada por todo el personal que labora en el servicio y los estudiantes..." (Virgo-E)

"El Código de Ética de Enfermería; bueno acá nosotros contamos con eso nada más, en algunos otros lugares el Colegio de Enfermeras, que nos puede prestar algún tipo de ayuda, desconozco otros." (Piscis-E)

Existe diversidad de documentos éticos-legales que respaldan el secreto profesional, pero se evidenció en los discursos que las enfermeras solo conocen algunos documentos como son; la historia clínica; Código de Ética y Deontología del Colegio Enfermeros del Perú; el consentimiento informado; además tienen referencia de otros documentos que respaldan el actuar de enfermería.

Cabe señalar, que la historia clínica es un documento privado, tiene carácter legal y su adulteración constituye falta punible por la ley, por lo que deberían numerarse las hojas de la historia para evitar problemas relacionados con la ley. ${ }^{15}$

El paciente es quien autoriza la divulgación de información asociada a su enfermedad. Sin embargo, excepcionalmente, por razones clínicas, de salud pública o judicial, su contenido podrá ser conocido, en forma reservada, por terceros. 16
Ética y legalmente, los pacientes tienen derecho a conocer el contenido de su historia clínica. Legalmente, el registro en sí pertenece al profesional o a la institución, pero la información contenida en el mismo pertenece a ellos. Los profesionales deben de conservar el original del registro y atender la solicitud del paciente con copias, a menos que la ley exija el registro original. A fin de proteger la confidencialidad, la información debe darse a conocer únicamente con el permiso por escrito del paciente o de su representante autorizado legal ${ }^{16}$.

Otro documento legal y primordial en el ejercicio profesional que refieren los sujetos de la investigación, es el Código de Ética y Deontología del Colegio Enfermeros del Perú; este código está constituido por un conjunto principios, normas, directivas y deberes que orientan y encauzan el ejercicio profesional de la Enfermera(o) Peruana(o). Por tanto, el profesional de enfermería, debe tener pleno conocimiento de este, ya que proporciona una sólida base para la orientación de sus acciones. En tal sentido, la enfermera tiene que considerar al hombre como un ser biopsicosocial y espiritual en constante interacción con su medio ambiente; la salud como un derecho y un deber atendiendo tanto las necesidades inmediatas, como aquellas que conlleven al mejoramiento de calidad de vida, en un clima de equidad, solidaridad, justicia y democracia. 17

También encontramos al consentimiento informado, que es la manifestación voluntaria, libre y racional realizada por un paciente, de aceptación a un tratamiento, luego de haber sido informado del mismo ya de habérsele respondido todas sus dudas de manera adecuada y suficiente, por ende se constituye en una exigencia ética de los derechos del ciudadano y está reconocido en la Ley General de Salud 2684217.

Está tiene las siguientes funciones: promover la autonomía de los individuos; fomentar la racionalidad en la toma de decisiones médicas; proteger a los enfermos y a los sujetos de experimentación; evitar el fraude y la coacción; alentar a la auto evaluación y auto examen de los 
profesionales de la salud; disminuir recelos y aliviar temores e introducir en la medicina una mentalidad más probabilística y más capaz de hacer frente a la incertidumbre.18,19 A su vez está basada en dos pilares: la confianza y el proceso de información más o menos complejo, esta última tendría que favorecer la comprensión del procedimiento propuesto, decir en qué consiste, las alternativas posibles, la oportunidad y los resultados esperados (beneficios, secuelas $y$ riesgos), con el fin de llegar a una decisión, sea de elección, de aceptación o de rechazo. ${ }^{19}$

\section{Conclusiones}

Después de haber realizado el análisis y discusión de la investigación referente al secreto profesional en el cuidado enfermero, se plantea las siguientes consideraciones finales:

- El secreto profesional es una confidencia que expresa el paciente a la enfermera, quien está comprometida a no divulgar y guardar la información que enmarca la vida íntima del ser cuidado.

- Las enfermeras se sienten en la obligación de revelar el secreto siempre y cuando esté en peligro la vida de la persona, de terceros y del equipo de salud.

- La divulgación del secreto profesional no se origina por parte de las enfermeras de los servicios de hospitalización que fueron escenario de estudio, sino por parte del personal profesional de Enfermería de otro servicio y del técnico de enfermería.

- Los profesionales de enfermería en su desempeño laboral, en los servicios de hospitalización, a diario se enfrentan a dilemas éticos, por las confidencias que reciben de los pacientes que se encuentran bajo su cuidado y en estos casos actúa en base a principios y valores que rigen su profesión.

- Respecto a los documentos ético legales que respaldan el secreto profesional, se evidenció escaso conocimiento por parte de las enfermeras, lo que origina dificultad en la práctica del secreto profesional.

\section{Bibliografía}

1. Pineda A.M. Conocimiento Sobre Aspectos éticos Legal del Personal de Enfermería que Labora en la Unidad de Emergencia del Hospital Central Universitario; 2003: [Consultada el 18 de octubre del 2012].Disponible en: bibmed.ucla.edu.ve/Edocs_bmucla/.../TIWY85G8 82003.pdf

2. Ángel Monge M. Ética Salud y Enfermedad. Madrid: Ediciones Palabra; 1991.

3. Instituto de Salud MSC "CRISTÓFORIS DÉNEKE". Temas Actuales de Ética y Bioética. Vol. 2. Lima: ISDEN; 2004.

4. Aranda Moreno L. Los Paradigmas de la Investigación Científica. Chiclayo: Universidad Católica Santo Toribio de Mogrovejo; 2000.

5. Vitores, A. Curso de Investigación Cualitativa: Fundamentos Técnicas y Métodos. España.

Antalya; 2004. [Consultada el Marzo 01 del 2013]. Disponible en:

http://antalya.uab.es/lioniuguez/aula/ic_analisi $s$ información.pdf.

6. Antón Almenara P. Enfermería. Ética y Legislación en Enfermería. Madrid: MASSON; 2003.

7. Beauchamp T.L., Childress J.F., Principios de la Ética Biomédica. Barcelona: MASSON; 1999.

8. Delgado Marroquín T. Confidencialidad y Secreto Profesional. Zaragoza. [Consultada el 17 de octubre del 2012]. Disponible en: www.institutodebioetica.org/.../...

9. Derechos Humanos. Actualizada: 23 de Junio 2012. [Consultada el 24 de Junio del 2011]. Disponible en: http:/ /www.indetectable.org/pages/historiaclin. 
htm

10. Villalobos M. El Secreto Profesional y la Confidencialidad de los Exámenes. [Consultada el 17 de octubre del 2012]. Disponible en: http://mariovillalobos.bligoo.com/content/vie w/ 392877/el-secreto-profesional-y-laconfidecialidad-de-los-examenes.html

11. Brunner y Suddarth. Enfermería Médico Quirúrgica. México: McGraw-HillInteramericana; 2005.

12. Velasco M.L, Fernández J., Arche Cono, Bonilla J., Cabrera E., Morales G. y Santamaría. Manual de Urgencias Médicas. Guía para Enfermeros y Paramédicos. Madrid: Cultural; 2008.

13. Castillo Córdova G. Ética y Educación en Valores. Perú: Universidad de Piura; 2004.

14. [Consultada el 23 de mayo del 2012]. Disponible en:

http://www.buenastareas.com/ensayos/DilemaEticos-Profesionales/353077.html

15. Fernández Vázquez J.M. Secreto Profesional. Volumen 44. México. Marzo de 1999. [Consultada el 16 de octubre del 2012]. Disponible en: http://www.medigraphic.com/pdfs/abc/bc- 1999/bc991h.pdf

16 .Del Río Sánchez C. Guía de Ética Profesional en Psicología Clínica. Madrid: PIRÁMIDE; 2011.

17. Código de Ética y Deontología del Colegio de Enfermeros del Perú. Perú. [Actualizada 1978.

Consultado el 26 de agosto del 2011 .].

Disponible en:

ftp://200.60.1 10.5/Docentes/Dpto_Enfermeria/ Raquelita_Diaz/sesion\%2010/Codigo\%20de\% 20Etica\%20y\%20Deontologia\%20del\%20Per \%FA.pdf

18. Zárate Cárdenas E. Los Derechos de los Pacientes y el Consentimiento Informado en el Perú. Perú. [Consultada el 17 de octubre del 2012]. Disponible en:

sisbib.unmsm.edu.pe/bvrevistas / situa/2004_n2 3/enpdf/a03.pdf.

19. Zárate C.E.; Cornejo R. Y Carhuapoma A.M. Los Profesionales de Salud y el Consentimiento Informado: Retos de la Educación Universitaria. Perú: 2006. [Consultada el 16 de octubre del 2012]. Disponible en:

sisbib.unmsm.edu.pe/bvrevistas/situa/2006_n 12 /pdf/a03.pdf 